\title{
Manipulation of optical patterns by frequency detuning of the pump beams
}

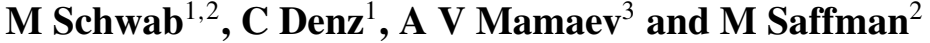 \\ ${ }^{1}$ Institute of Applied Physics, Darmstadt University of Technology, Hochschulstr. 6, \\ 64289 Darmstadt, Germany \\ 2 Department of Physics, University of Wisconsin, 1150 University Avenue, Madison, \\ WI 53706, USA \\ ${ }^{3}$ Institute for Problems in Mechanics, Russian Academy of Sciences, Prospekt Vernadskogo \\ 101, Moscow, 117526 Russia
}

Received 15 December 2000, in final form 20 August 2001

Published 18 September 2001

Online at stacks.iop.org/JOptB/3/318

\begin{abstract}
We show that manipulation and selection of optical patterns is possible by detuning the frequency of one of the two waves in counterpropagating photorefractive two-wave mixing with a feedback mirror. An analytical expression for the onset of modulational instability is derived for the general case of an arbitrary complex coupling constant, including a possible frequency shift of the generated sidebands. The influence of frequency detuning of the pump beams on the spatial scale of the resulting pattern and on the threshold value of the photorefractive coupling strength for modulational instability is investigated. The spatial scale and the frequency detuning of the generated sidebands were measured in an experiment using a photorefractive $\mathrm{KNbO}_{3}$ crystal. Comparison of theory and experiment show agreement for positive frequency detuning of the pump beams. For negative detuning we observe clamping of the spatial scale and no frequency shift of the sidebands. Possible explanations of this deviation between analytical results and experiment are discussed.
\end{abstract}

Keywords: Nonlinear optics, pattern formation, transverse effects, photorefractive media

\section{Introduction}

The spontaneous formation of spatial patterns in nonlinear optical materials has been studied intensively in the last few years $[1,2]$. Despite the differences in nonlinearities, various media such as atomic vapours [3], liquid crystals [4], liquid crystal light valves [5], and photorefractive crystals [6-8] show similar spatial patterns in the presence of optical feedback, and are therefore excellent model systems for extensive studies of pattern formation effects. Photorefractive materials like $\mathrm{KNbO}_{3}$ and $\mathrm{BaTiO}_{3}$ have especially proven to be an excellent choice, since they provide large nonlinearities at low laser powers in the milliwatt-range and constitute alloptical systems without any external power supply. The control parameters are easily accessible and quantitative measurements can be performed in real time. They also show a great variety of different pattern states, among them hexagons, squeezed hexagons, squares, rectangles, rhomboids or stripes, depending on the position of the feedback mirror and its reflectivity $[8,9]$. In particular, when an imaging system produces a virtual feedback mirror inside the crystal, a region of multiple pattern stability was recently reported [9] yielding a large number of possible states for the same set of external parameters. To date, theoretical investigations of photorefractive pattern formation have been based on linear stability analysis, as in [7,8], and on a nonlinear analysis showing the dominance of a hexagonal pattern for small distances of the feedback mirror from the medium [10]. In the realm of possible technological applications [11], great attention has recently been paid to the manipulation, stabilization and control of these spontaneous patterns, using spectral control techniques manipulating the optical feedback in order to force the system to a desired solution [12-14], or to stabilize otherwise unstable pattern states $[15,16]$.

In this article we demonstrate the successful experimental manipulation of a pattern state by frequency-detuning one 


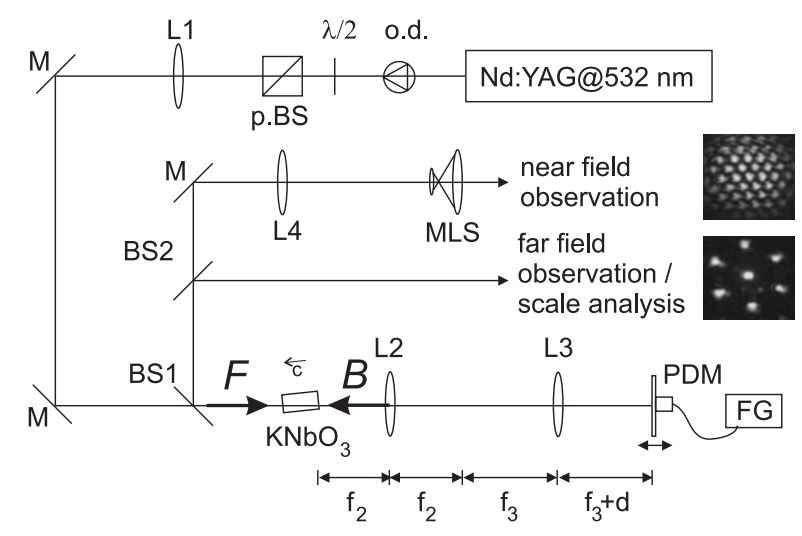

Figure 1. Experimental set-up used for pattern observation and measurements: O.d. $=$ optical diode (Faraday rotator); $\lambda / 2=$ halfwave plate; $\mathrm{p} . \mathrm{BS}=$ polarizing beam splitter; $\mathrm{BS}=$ beam splitter; $\mathrm{M}=$ mirror; $\mathrm{L}=$ lens; $\mathrm{PDM}=$ piezo driven mirror; $\mathrm{FG}=$ frequency generator; $\mathrm{MLS}=$ microscope lens system. Also shown is the direction of the $c$-axis (direction of energy transfer) of the photorefractive $\mathrm{KNbO}_{3}$ crystal.

of the two beams interacting in a photorefractive medium. Due to the sluggish response of the photorefractive effect, a frequency detuning of the interacting optical beams changes both the amplitude and phase of the complex coupling constant. This affects the spatial symmetry and the transverse scale of the pattern, as well as the threshold value of the photorefractive coupling strength. In order to model this behaviour we have extended previous theoretical treatments of modulational instability in photorefractive feedback mirror experiments. Here we allow for frequency detuning of one of the pump beams as well as for an additional frequency detuning of the spatial sidebands. The experiments are performed with samples of photorefractive $\mathrm{KNbO}_{3}$, which has been the material of choice for the majority of pattern formation experiments using photorefractive materials. The charge transport in $\mathrm{KNbO}_{3}$ is diffusion-dominated which implies an imaginary coupling constant and strong energy transfer in photorefractive two-beam coupling.

The rest of this paper is organized as follows. In section 2 the experimental set-up together with observations of pattern selection and scale changes with frequency shifted pump beams are discussed. A linear stability analysis with frequency shifted pump beams is performed in section 3 . The resulting dispersion relation is used to calculate the threshold nonlinearity, spatial scale, and sideband frequency shift for a range of experimentally relevant parameters. In section 4 we compare measurements of spatial scale and sideband frequency with the predictions of the linear stability analysis. The paper concludes with section 5 where we discuss the qualitatively different results for positive and negative frequency detuning.

\section{Experimental results}

The experimental arrangement is shown in figure 1 . The beam from a frequency-doubled Nd:YAG laser operating at a wavelength of $532 \mathrm{~nm}$ was sent through an optical diode and a variable attenuator consisting of a half-wave plate and a polarizing beam splitter. It was then focused by a lens with a focal length of $f_{1}=501 \mathrm{~mm}$ into the photorefractive crystal (beam diameter about $300 \mu \mathrm{m}, P=12 \mathrm{~mW}$ incident on the crystal). We used two crystal samples with $l_{1}=$ $3.4 \mathrm{~mm}$ and $l_{2}=5 \mathrm{~mm}$ along the crystallographic $c$-axis providing sufficient coupling gain to access the multiple pattern region [9]. The crystals were oriented in a way that the incoming beam, denoted here as $F$ (forward), was depleted, the feedback beam $B$ (backward) being amplified. The laser beam was polarized parallel to the crystallographic $a$-axis to take advantage of the large $r_{13}$-coefficient of the electro-optic tensor in $\mathrm{KNbO}_{3}$ [17]. The optical feedback was provided by a confocal system of two lenses with focal lengths of $f_{2}=150 \mathrm{~mm}$ and $f_{3}=200 \mathrm{~mm}$, respectively, and a mirror which was placed at a distance $d$ from the 1:1 imaging position. The feedback system creates a virtual mirror at a distance of $L=\left(f_{2} / f_{3}\right)^{2} d$ from the rear crystal surface, as can be shown using beam transfer matrices [18]. In addition, negative virtual mirror positions could be achieved which is an essential experimental tool to access the multiple pattern region [9]. The mirror was placed on a translation stage for controlled adjustment and measurement of the diffraction length $d$, and hence $L$. The motion of the feedback mirror was controlled by mounting it on a piezo actuator connected to a frequency generator producing the desired voltage ramps to impress a frequency detuning on the feedback beam. Above a certain threshold, this system gives rise to a modulational instability, leading to spatial sidebands in the optical far field, both in the forward and backward direction. These sidebands can be monitored in the far field (power spectrum) and by means of a lens and a microscope lens system in the near field producing an actual image of the Gaussian laser beam filamented into an hexagonal array (shown in figure 1 for $L=0$ ).

To investigate the effects of a frequency detuning on the pattern state, a diffraction length of about $n_{0} L / l=$ $-0.30 \pm 0.10$ was chosen, which is inside the multiple pattern region. Using a circular spatial filter between the two lenses in the feedback to suppress instabilities with a larger transverse wave number, a square pattern was achieved as the stable solution of the system as depicted in figure 2(a). By applying a small frequency shift to the feedback beam, $\Omega=2.78$ $\mathrm{rad} \mathrm{s}^{-1}$, it was possible to switch to a hexagonal pattern with a smaller transverse wave number (figure $2(b)$ ). Turning off the frequency detuning, the system relaxed back to the square type solution. A whole sequence of transitions between hexagons and squares could be performed by switching the frequency generator on and off. The switching time was some tens of milliseconds which corresponds to the relaxation time of the photorefractive grating at the intensity of approximately $4 \mathrm{~W} \mathrm{~cm}^{-2}$ used for this experiment. For a different diffraction length, $n_{0} L / l=-0.60 \pm 0.10$, where squeezed hexagons were found to be the stable solution, a switch from the squeezed hexagon to a rectangular solution could be performed, using the same frequency detuning $\Omega=2.78 \mathrm{rad} \mathrm{s}^{-1}$. It was also possible to continuously switch between a hexagonal and a square pattern by applying a triangular voltage yielding a frequency shift of $\Omega= \pm 4.35 \mathrm{rad} \mathrm{s}^{-1}$ for a mirror position of $n_{0} L / l=-0.35 \pm 0.10$. In a region of parameters where the hexagonal pattern is known to be the only solution the system selects (outside the multiple pattern region), a change to a different pattern could not be obtained. Here, a frequency shift only affected the transverse wave number of the hexagon. 

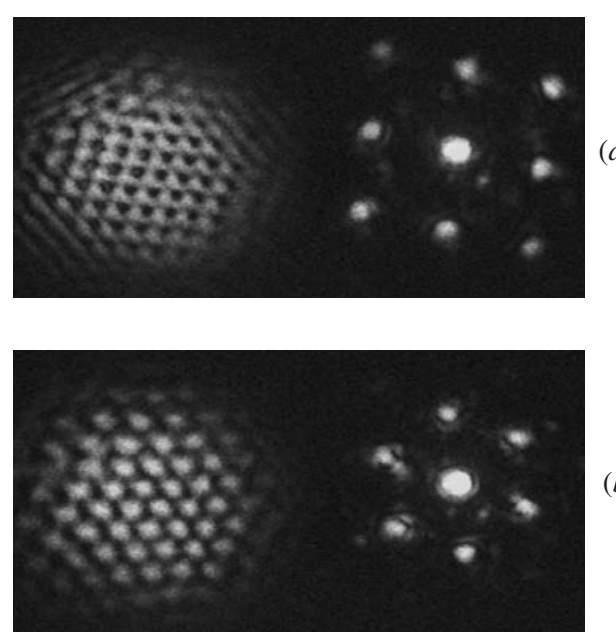

(b)
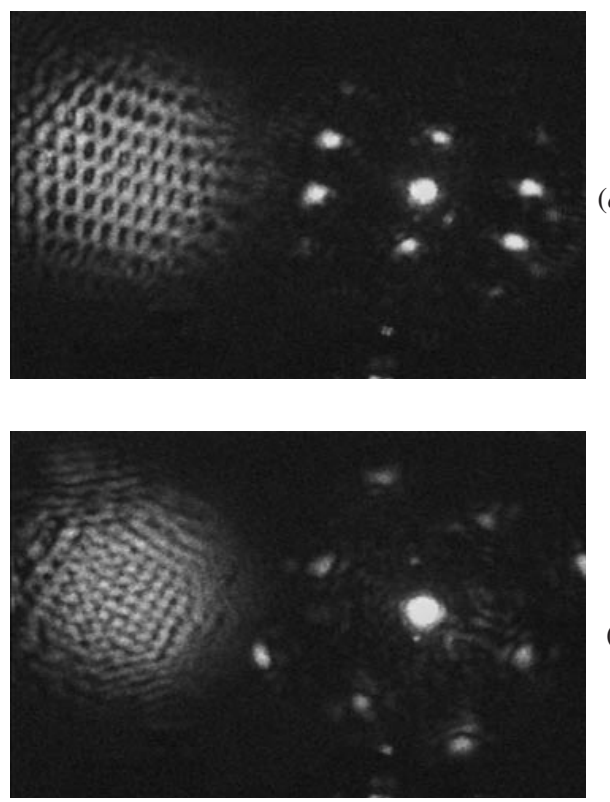

$(d)$

Figure 2. Pattern switch obtained by introducing a frequency detuning to the beam reflected from the feedback mirror. (a) Square pattern state without frequency detuning, $\Omega=0$, and $n_{0} L / l=-0.30$. Measured transverse wave number $k_{d} l=3.5$. (b) Controlled hexagonal pattern state with frequency detuned pump beams at $\Omega=2.78 \mathrm{rad} \mathrm{s}^{-1}$, yielding a smaller transverse wave number of $k_{d} l=2.7$. (c) Squeezed hexagonal pattern state without frequency detuning, $\Omega=0$, and $n_{0} L / l=-0.60$ on two different transverse wave numbers. $(d)$ Controlled rectangular pattern state with frequency detuned pump beams, $\Omega=2.78 \mathrm{rad} \mathrm{s}^{-1}$.

We performed measurements of the transverse wave number as a function of the frequency detuning for different values of $n_{0} L / l$. A slit filter was introduced to make the optical feedback basically one-dimensional as in the theoretical treatment presented in section 3 . The pattern appearing for this configuration is a spatial roll pattern $[8,12]$ whose transverse angular extension $\theta$ can easily be measured. The transverse wave number can then be directly calculated using $k_{d} l=$ $\pi \theta^{2} l /\left(\lambda n_{0}\right)$. Results of these measurements are depicted in figure 3 and show the asymmetry regarding the pump frequency detuning $\Omega$. We found experimentally that the spatial scale of the pattern stayed basically constant for negative frequency detunings, while an increase of $\Omega$ in the positive range leads to

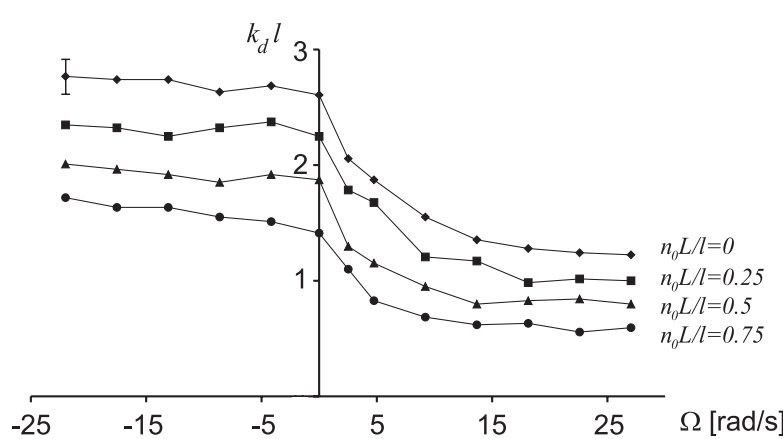

Figure 3. Experimentally obtained transverse wave number as a function of the frequency detuning for different values of $n_{0} L / l$. The points are linearly connected to guide the eye.

a decreasing value of the transverse wave number in the optical far field (i.e. an increasing spatial period in the optical near field). The curves for different values of the diffraction length $n_{0} L / l$ are well-separated from each other, and all curves show the same qualitative behaviour. For the range of parameter values considered here $\left(n_{0} L / l \geqslant 0\right)$, the two-dimensional case gave similar results, with the hexagonal pattern showing a decrease of size for larger positive $\Omega$, and no significant reaction to a negative frequency shift $\Omega$.

This fundamental asymmetry in the sign of the frequency shift of the pump beams was also apparent on closer inspection of the temporal behaviour of the spatial sidebands. For positive frequency detuning $\Omega$, we found an oscillation of the sideband intensity, with dark fringes and vortices carrying phase dislocations which move through the spatially extended sidebands. Snapshots of the intensity distribution of a spatial sideband reveal situations with one or more vortices, chosen examples with one and four vortices can be seen in figure 4 and show the similarity of these pictures to transverse modes obtained in photorefractive oscillators [19]. The accompanying interferograms in figure 4 clearly indicate the existence of phase singularities in the optical beam, for the case of a positive frequency detuning $\Omega$. Negative frequency detunings $\Omega$, on the other hand, did not result in significant temporal variations of the sideband intensity. Additionally, we also performed measurements of the sideband frequency detuning $\delta$ by beating the sideband with the pump beam directly derived from beam splitter BS1 in figure 1 and measuring the beat frequency. We found experimentally that the frequency detuning $\delta$ coincides with the frequency of the intensity oscillations for positive $\Omega$, while negative $\Omega$ did not result in any significant sideband frequency detunings $\delta$.

To validate the proper functioning of our mirror, we performed an interferometric measurement of the beat frequency between the pump beam and the beam reflected from the piezo mirror and found no hints of an asymmetric behaviour for different directions of motion of the mirror. Thus, the experimentally found properties and asymmetries are inherent to the pattern forming system. The asymmetric results for positive and negative frequency detunings will be addressed below in comparison with the corresponding theoretical curves. 
Manipulation of optical patterns by frequency detuning of the pump beams
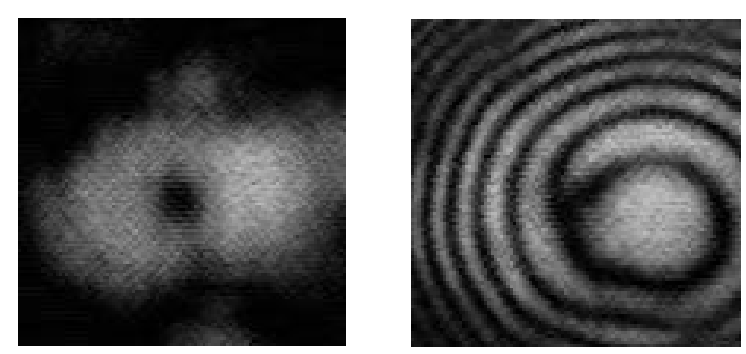

(a)

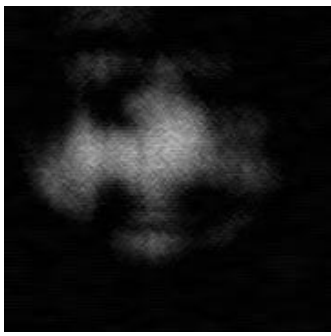

Intensity

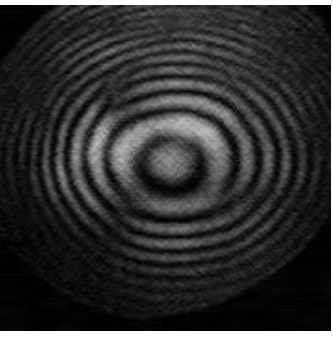

Interferogram
Figure 4. Snapshots of the sideband intensity profiles taken for $L=0$ and a frequency detuning of $\Omega=4.75 \mathrm{rad} \mathrm{s}^{-1}$. (a) One vortex, and $(b)$ four vortices appearing in the spatial sideband. Left: intensity pattern; right: interferogram of the sideband with a coherent spherical wave.

\section{Linear stability analysis}

In order to understand the effect of a frequency detuning on the pattern forming process, we performed a linear stability analysis extending earlier results [7]. The paraxial equations of motion for counterpropagating two-wave mixing including diffraction and grating dynamics can be written as $[7,20]$

$$
\begin{gathered}
\frac{\partial F}{\partial z}-\frac{i}{2 k_{0} n_{0}} \nabla_{\perp}^{2} F=g B \\
\frac{\partial B}{\partial z}+\frac{i}{2 k_{0} n_{0}} \nabla_{\perp}^{2} B=g^{\star} F \\
\left(\tau \frac{\partial}{\partial t}+1\right) g=i \gamma \frac{F B^{\star}}{|F|^{2}+|B|^{2}}
\end{gathered}
$$

where $F$ and $B$ denote the amplitudes of the two counterpropagating beams, $k_{0}=2 \pi / \lambda$ is the wave number of the laser light in vacuum, $n_{0}=2.33$ denotes the linear refractive index of the crystal, $\nabla_{\perp}$ is the transverse Laplacian and $g$ denotes the amplitude of the photorefractive index grating. $\tau$ is the relaxation time of the photorefractive medium, and $\gamma$ is a measure for the photorefractive nonlinearity and constitutes a measure for the amplitude and phase transfer in the photorefractive two-wave mixing process. $\gamma=\gamma_{R}+i \gamma_{I}$ is generally a complex value and depends on the strength of the refractive index modulation, the wavelength of the incident beams and the relative phase shift of the refractive index grating with respect to the interference pattern of the incident waves [17]. We assume an externally imposed frequency detuning of the pumps, denoted here as $\Omega$, and allow for an additional spontaneous frequency detuning $\delta$ of the spatial sidebands. The perturbation ansatz therefore reads as

$$
F(z)=\bar{F}_{0}(z)\left\{1+f_{+} \mathrm{e}^{\mathrm{i}\left(\vec{k}_{\perp} \cdot \vec{r}-\delta t\right)}+f_{-} \mathrm{e}^{-\mathrm{i}\left(\vec{k}_{\perp} \cdot \vec{r}-\delta t\right)}\right\}
$$

$$
B(z)=\bar{B}_{0}(z)\left\{1+b_{+} \mathrm{e}^{\mathrm{i}\left(\vec{k}_{\perp} \cdot \vec{r}-\delta t\right)}+b_{-} \mathrm{e}^{-\mathrm{i}\left(\vec{k}_{\perp} \cdot \vec{r}-\delta t\right)}\right\}
$$

with the plane wave solutions

$$
\begin{aligned}
& \bar{F}_{0}(z)=F_{0}(z) \mathrm{e}^{\mathrm{i}\left(k_{0} z-\omega_{0} t\right)} \\
& \bar{B}_{0}(z)=B_{0}(z) \mathrm{e}^{\mathrm{i}\left(-k_{0} z-\omega_{0} t\right)} \mathrm{e}^{-\mathrm{i} \Omega t}
\end{aligned}
$$

where $w_{0}=2 \pi c / \lambda$ denotes the optical frequency of the incident light. The backward pump beam $B$ carries the additional frequency detuning $\Omega$. Inserting equations (4) and (5) into the two-wave mixing equations (1), (2) and (3), four equations for the four unknown amplitudes $f_{+}, f_{-}^{\star}, b_{+}$and $b_{-}^{\star}$ are obtained (see [7] or [21] for details of this calculation). Splitting $\gamma$ into its real and imaginary part, $\gamma=\gamma_{R}+i \gamma_{I}$, the analytical threshold condition for modulational instability in the case of counterpropagating two-wave mixing with mirror boundary conditions and a complex coupling constant reads as $\cos \left(w_{1} l\right) \cos \left(w_{2} l\right)$

$$
\begin{aligned}
& +\frac{\sin \left(w_{1} l\right) \sin \left(w_{2} l\right)}{2 w_{1} w_{2}}\left(\eta+\mu \cos \left(2 k_{d} n_{0} L\right)+v \sin \left(2 k_{d} n_{0} L\right)\right) \\
& +\frac{\sin \left(w_{1} l\right) \cos \left(w_{2} l\right)}{2 w_{1}} \chi_{0}\left(\gamma_{I} \cos \left(2 k_{d} n_{0} L\right)-\gamma_{R} \sin \left(2 k_{d} n_{0} L\right)\right) \\
& -\frac{\cos \left(w_{1} l\right) \sin \left(w_{2} l\right)}{8 w_{2}}\left(\zeta \cos \left(2 k_{d} n_{0} L\right)+\rho \sin \left(2 k_{d} n_{0} L\right)\right)=0
\end{aligned}
$$

with the abbreviations

$$
\begin{aligned}
k_{d} & =\frac{k_{\perp}^{2}}{2 k_{0} n_{0}} \\
w_{1} & =\sqrt{k_{d}^{2}+k_{d} \chi_{0} \gamma_{R}-\frac{1}{4} \chi_{0}^{2} \gamma_{I}^{2}} \\
w_{2} & =\sqrt{k_{d}^{2}+\frac{1}{4} k_{d} \rho-\frac{1}{64} \zeta^{2}}
\end{aligned}
$$

$$
\begin{aligned}
& \eta=-\frac{1}{8} \chi_{0} \gamma_{I} \zeta+2 k_{d}^{2}+\chi_{0} \gamma_{R} k_{d}+\frac{1}{4} \chi_{0} \gamma_{R} \rho+\frac{1}{4} k_{d} \rho \\
& \mu=-\chi_{0} \gamma_{R} k_{d}-\frac{1}{4} \chi_{0} \gamma_{R} \rho-\frac{1}{4} k_{d} \rho \\
& v=\frac{1}{8} \chi_{0} \gamma_{R} \zeta+\frac{1}{4} k_{d} \zeta-\chi_{0} \gamma_{I} k_{d}-\frac{1}{8} \chi_{0} \gamma_{I} \rho
\end{aligned}
$$

and

$$
\begin{array}{r}
\rho\left(\gamma_{I}, \gamma_{R}, \Omega, \delta\right)=2 \gamma_{R}\left[\left(\chi_{0}+\chi_{0}^{\star}\right)-\left(\chi_{-}+\chi_{+}^{\star}\right)\right] \\
+2 i \gamma_{I}\left[\left(\chi_{0}-\chi_{0}^{\star}\right)-\left(\chi_{-}-\chi_{+}^{\star}\right)\right] \\
\zeta\left(\gamma_{I}, \gamma_{R}, \Omega, \delta\right)=2 i \gamma_{R}\left[\left(\chi_{0}-\chi_{0}^{\star}\right)-\left(\chi_{-}-\chi_{+}^{\star}\right)\right] \\
-2 \gamma_{I}\left[\left(\chi_{0}+\chi_{0}^{\star}\right)-\left(\chi_{-}+\chi_{+}^{\star}\right)\right]
\end{array}
$$

with

$$
\begin{aligned}
\chi_{0} & =\frac{1}{1+i \Omega \tau}, \\
\chi_{ \pm} & =\frac{1}{1+i(\Omega \pm \delta) \tau} .
\end{aligned}
$$

Equation (8) can be checked for the special case $\Omega=0$ and $\delta=0$ against the equation given in [7]. For a purely real 

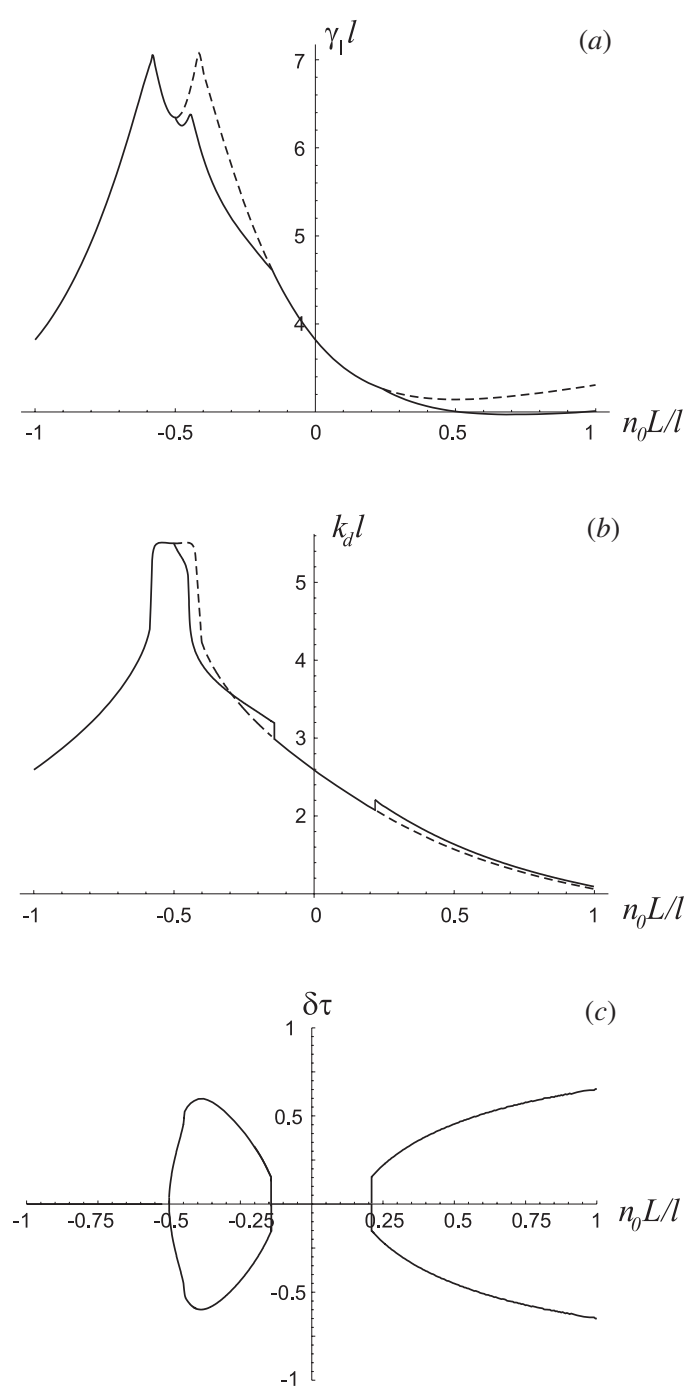

Figure 5. Threshold curves for linear instability as a function of the normalized mirror position $n_{0} L / l$ for the special case $\Omega=0$. (a) Threshold coupling strength $\gamma_{I} l ;(b)$ corresponding transverse wave number $k_{d} l$ and $(c)$ sideband frequency detuning $\delta \tau$. Solid curve: values connected to the absolute minimum of the threshold curves $\gamma_{I} l\left(k_{d} l\right)$; dashed curve: curves connected to a static instability $\delta=0$, but larger threshold value $\gamma_{I} l$.

coupling constant, $\gamma_{I}=0$, the equation is also consistent with the expression given in [22] for the case of a drift-dominated photorefractive material. To compare our theoretical results with the experimental measurements, we will now concentrate on the case of a purely imaginary coupling constant, $\gamma=i \gamma_{I}$, which represents the experimentally relevant case of $\mathrm{KNbO}_{3}$. For each value of the mirror position $L$ and the frequency detuning $\Omega$, one can calculate the threshold function $\gamma_{I}\left(k_{d} l\right)$ from equation (8) (see the corresponding special cases for $\Omega=0$ and different values of $L$ in $[7,8,10]$ as examples). The assumption of an additional frequency detuning $\delta$ in the spatial sidebands extends the earlier analyses, also for the case of $\Omega=0$. Allowing for solutions with nonzero $\delta$, branches with lower threshold values of the photorefractive coupling strength can be found thus modifying these earlier results.

For the special case of $\Omega=0$, figure 5 shows the dependence of the minimal values of the threshold coupling
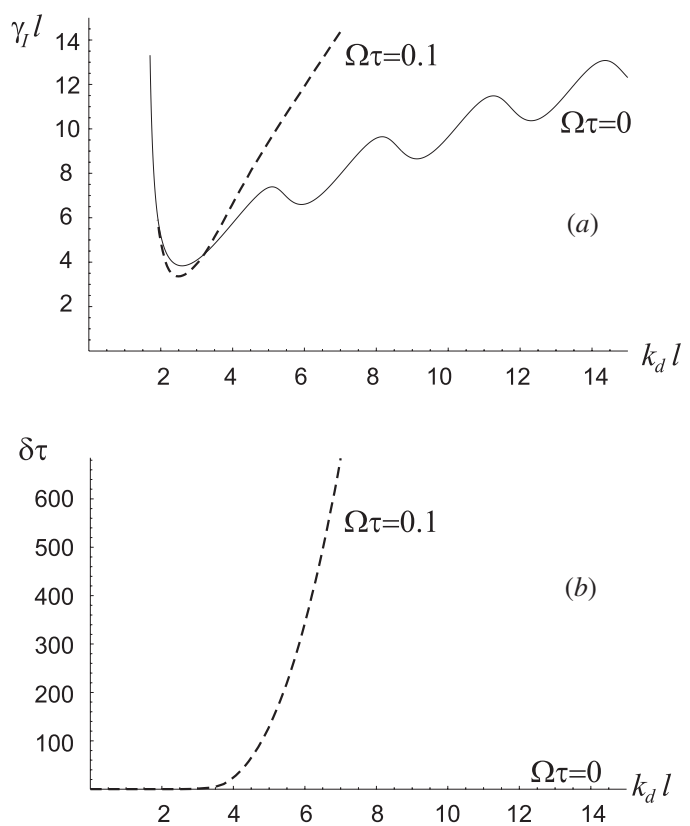

Figure 6. Calculated threshold curve for modulational instability as a solution of equation (8). (a) Coupling strength $\gamma_{I} l$ as a function of the transverse wave number for parameter value $L=0$ and $\Omega=0$ (solid curve), and $\Omega \tau=0.1$ (dashed curve), together with the values of the sideband frequency detuning $\delta \tau$ in $(b)$. The case of $L=0$, $\Omega=0$ represents a special case where no nonzero solutions of $\delta$ can be found.

strength $\gamma_{I} l$, the corresponding most unstable transverse wave number $k_{d} l$, and the sideband frequency detuning $\delta$ on the diffraction length $n_{0} L / l$. Here, the solid curves depict the absolute minima of the curves $\gamma_{I}\left(k_{d} l\right)$ allowing for solutions of the threshold condition with $\delta \neq 0$. Only for $n_{0} L / l \leqslant-0.5$ and the window $-0.14 \leqslant n_{0} L / l \leqslant 0.21$, do the absolute minima of the threshold curves correspond to the branch with $\delta=0$. The dashed curves represent the threshold curves for static instabilities, $\delta=0$ as found e.g. in $[8,10]$, but with a larger threshold value compared to the branches allowing for dynamic instabilities with $\delta \neq 0$. Even for this special case $\Omega=0$, an inclusion of an additional frequency detuning $\delta$ in the spatial sidebands is necessary to find the minimum value for the coupling strength $\gamma_{I} l$, where the system is unstable to perturbations with a specific transverse wave number $k_{d} l$. This constitutes an important feature of our extended analysis and was not taken into account in previous publications where only static instabilities $(\delta=0)$ were allowed. A nonzero $\Omega$ always requires a nonzero frequency detuning $\delta$ in the spatial sidebands to solve the threshold equation (8). Therefore, a threshold curve $\gamma_{I}\left(k_{d} l\right)$ is accompanied by a curve $\delta\left(k_{d} l\right)$ yielding a nonzero value at the corresponding critical wave number. Examples of such threshold curves are depicted in figure 6 for the case $L=0$ and two different values of $\Omega$. One can conclude from figure $6(a)$, that a positive frequency detuning lowers both the threshold value for modulational instability $\gamma_{I} l$ and the corresponding transverse wave number $k_{d} l$. The minimum of each particular curve $\gamma_{I}\left(k_{d} l\right)$ determines the threshold value for the photorefractive coupling strength $\gamma_{I}$, the corresponding transverse wave number $k_{d}$, which is a measure for the wavelength of the resulting pattern in the optical near field, and the frequency detuning $\delta$ of the spatial 


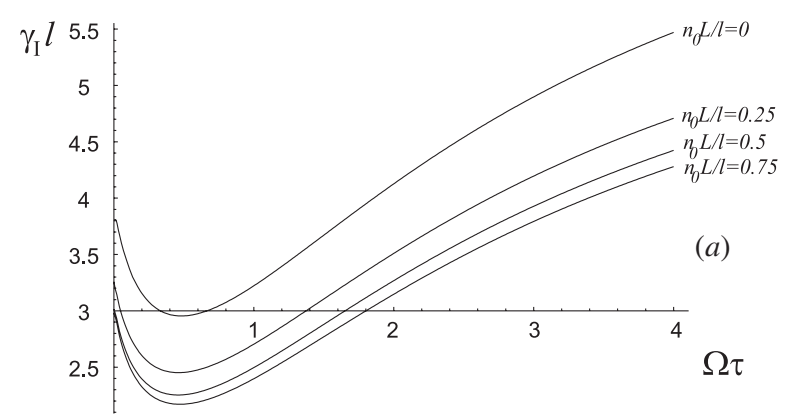

$\gamma_{I} l$
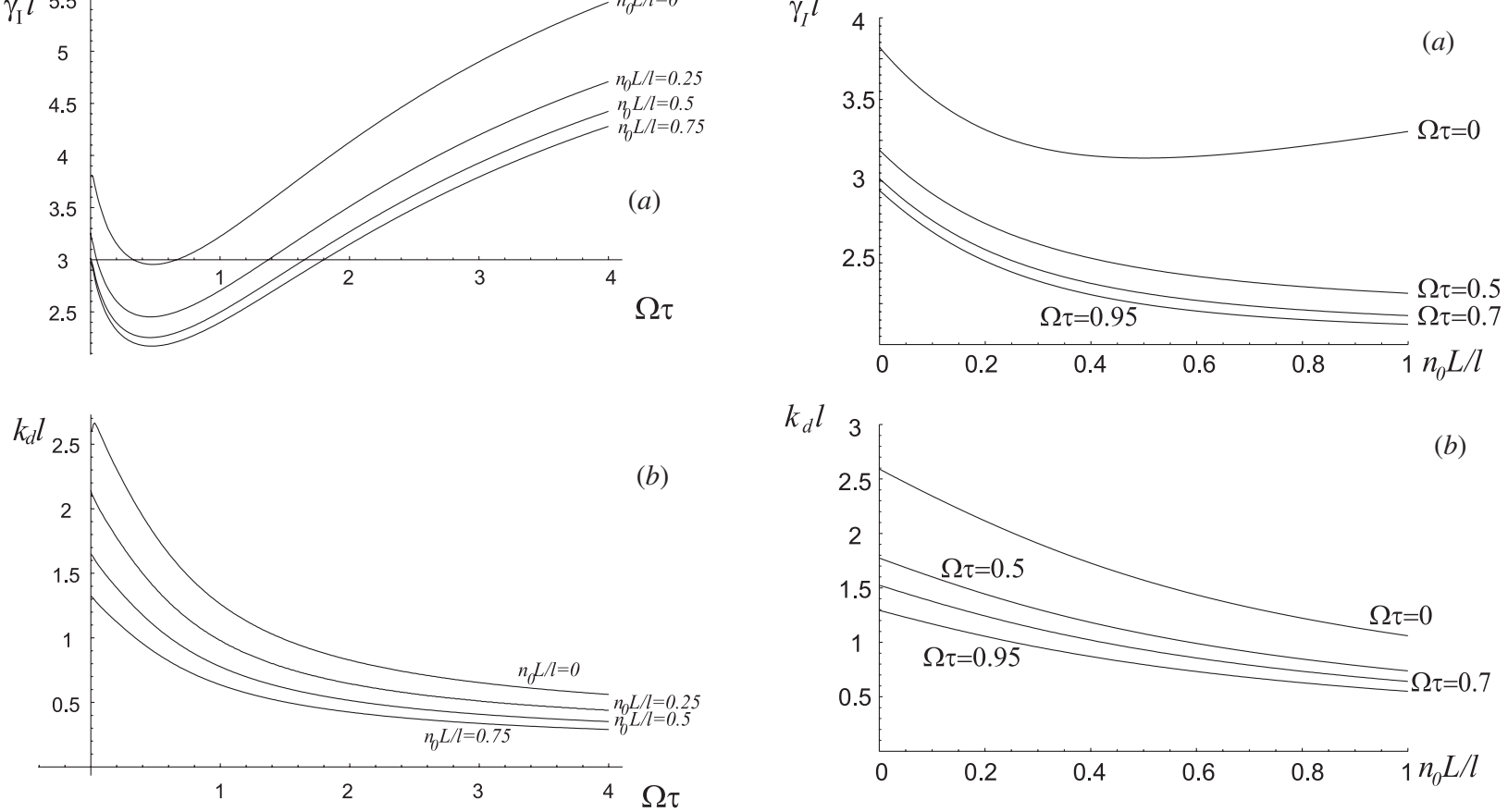

$n_{0} L / l=0.75$

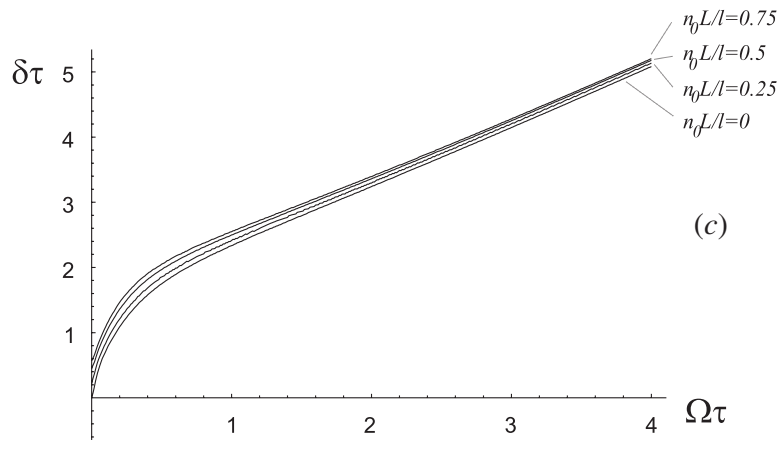

Figure 7. Threshold curves showing the minima of the curves $\gamma_{I} l\left(k_{d} l\right)$ as a function of the frequency detuning $\Omega \tau$. (a) Threshold coupling strength $\gamma_{I} l 0 ;(b)$ corresponding transverse wave number $k_{d} l$, and $(c)$ frequency detuning $\delta \tau$ in the sidebands for selected values of $n_{0} L / l$.

sidebands. Figure 7 shows the position of these minima as a function of the frequency detuning $\Omega \tau$ for selected values of the normalized mirror position $n_{0} L / l$. Figure $7(a)$ indicates that the threshold value for modulational instability is lowered for small positive frequency detunings $\Omega$. The shape of the curves for different values of $n_{0} L / l$ is identical, the only difference being an offset in $\gamma_{I} l$. The corresponding transverse wave numbers $k_{d} l$ decrease for larger frequency detunings $\Omega \tau$, whereas the frequency detuning $\delta \tau$ in the sidebands increases.

Additional analysis of equation (8) for certain negative values of $\Omega$ and $L \neq 0$ resulted in very large negative values of the sideband frequency shift. The reason for this is at present not understood and has not been observed in experiment. We have therefore concentrated on positive values of $\Omega$ in comparison of theory and experiment. However, for the special case $L=0$, the threshold curves could be calculated for both positive and negative values of $\Omega$, and the results will be discussed together with the experimental data for these parameters in section 4 . To complete the theoretical analysis,
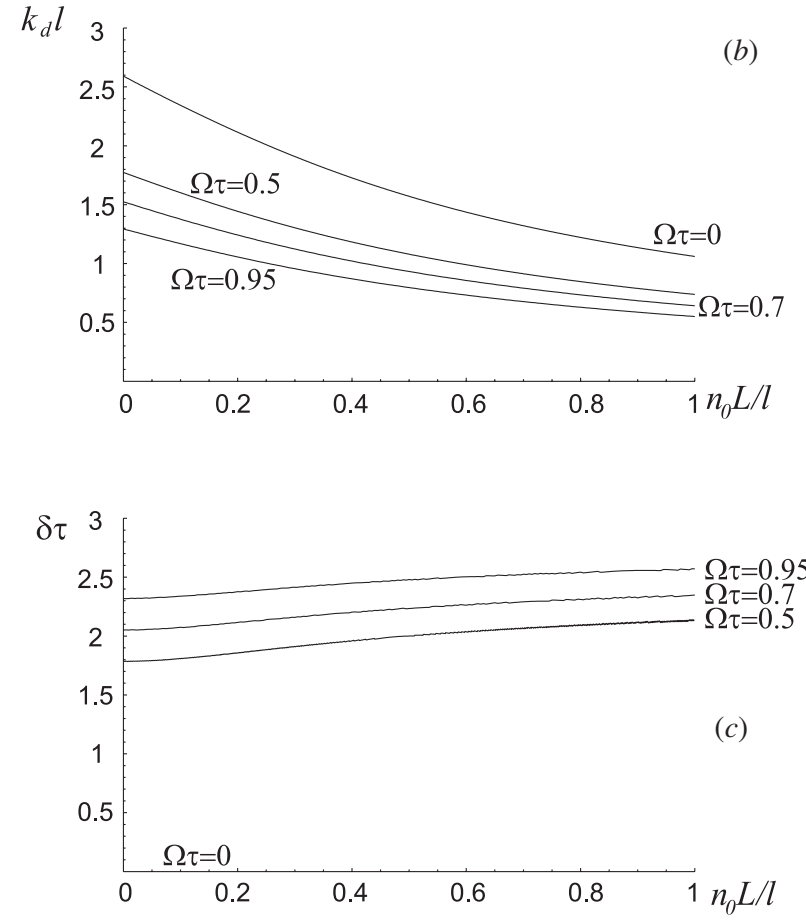

Figure 8. Threshold curves showing the minima of the curves $\gamma_{I} l\left(k_{d} l\right)$ as a function of the normalized propagation length $n_{0} L / l$. (a) Threshold coupling strength $\gamma_{I} l,(b)$ corresponding transverse wave number $k_{d} l$, and $(c)$ frequency detuning $\delta \tau$ in the sidebands for selected values of $\Omega \tau$.

figure 8 shows the threshold coupling strength, transverse wave number, and the corresponding frequency detuning of the sidebands as a function of the normalized diffraction length $n_{0} L / l$, for various values of $\Omega \tau$. It is worth noting that the qualitative results obtained here for mirror boundary conditions are similar to those reported for two frequency shifted external beams in [21]. For both boundary conditions, an inherent asymmetry concerning positive and negative values of the frequency detuning $\Omega$ persists. Small positive values of $\Omega$ lead to a reduced threshold coupling strength and a smaller spatial scale of the pattern in the optical far field. In both cases, nonzero sideband frequency detunings $\delta$ are required to solve the respective threshold condition.

\section{Comparison of experiment and theory}

The relaxation time constant $\tau$ of the photorefractive crystal is a key element in comparing the results of the linear stability analysis with experimental observations. Before performing 
this comparison, we must account for the indirect dependence of the time constant $\tau$ on the frequency detuning $\Omega$.

In photorefractive media the time constant is to a first approximation inversely proportional to the average intensity [17, 23], $\tau \sim I^{-1}=\left(|F|^{2}+|B|^{2}\right)^{-1}$. However, in the case of a reflection grating interaction between frequency shifted beams, the solution for the intensity depends on the coupling constant which in turn has the effective value

$$
\bar{\gamma}=\frac{\gamma}{1+i \Omega \tau}=\gamma \frac{1-i \Omega \tau}{1+\Omega^{2} \tau^{2}}
$$

where the overbar indicates the coupling constant in the presence of a frequency shift. The imaginary part of the coupling constant is thus changed to

$$
\bar{\gamma}_{i}=\frac{\gamma_{i}-\gamma_{r} \Omega \tau}{1+\Omega^{2} \tau^{2}}
$$

The reduced coupling constant as given in (20) has a direct effect on the energy coupling between the two counterpropagating beams and changes the average intensity in the crystal. Therefore when $\Omega \neq 0$ we can not solve analytically for the intensity inside the crystal and instead use the following iterative procedure.

Utilizing the analytical expressions for the $z$-dependences of the beam intensities $|F|^{2}$ and $|B|^{2}$ for $\Omega=0$ as given in [17] and assuming an intensity dependence of $\tau$ according to $\tau \sim I^{-1}[17,23]$, we compute the average intensity in the crystal and the time constant $\tau$. This $\tau$ then modifies $\bar{\gamma}$, which is used to find an improved estimate for the intensity. In an iterative process, we proceed until we reach a stable asymptotic value of the intensity and $\tau$. These values as a function of $\Omega \tau$ are depicted in figure 9 for a starting value of $\tau_{0}=75 \mathrm{~ms}$ and three values of $\gamma_{R}$ indicating a considerable and non-negligible effect. The other parameters used were $\gamma_{I} l=4$ and a net intensity reflectivity of the feedback system of $R \approx 0.5$ due to two Fresnel reflections at the crystal surface (transmission coefficient $T \approx 0.84$ and losses when passing the lenses) ${ }^{5}$. The value of $\tau$ as depicted in figure 9 is taken account of in the following analysis.

Figures 10(a)-(c) show examples of the results of the measurements of the transverse wave number as presented in section 2 together with the corresponding theoretical curves. Figure 10 $(a)$ shows the dependence of the transverse wave number $k_{d} l$ on the frequency detuning for $n_{0} L / l=0$ and a value of $\tau_{0}=75 \mathrm{~ms}$. The theoretical curves are given for three different drift components $\gamma_{R}$ of the photorefractive coupling constant. There is good agreement between theory and experiment for positive frequency detunings $\Omega$. Also the asymmetry of the experimental curve can be reproduced in theory, only the step in the transverse wave number for small negative frequency detunings $\Omega$ in theory does not appear in the experiment. This step is due to the existence of a competing minimum taking over the absolute minimum of the threshold coupling strength. The experimentally found

\footnotetext{
5 The calculation of the change in $\tau$ used $R=0.5$ to be in close agreement with the experimental values. However, assuming $R=1$ as was used in the linear stability analysis leads to a higher mean intensity level and as a consequence, a lower value of $\tau$, that differs by only $3 \%$ from the value found with $R \approx 0.5$ for $\Omega \tau=2$. Thus there is no significant inconsistency with the stability analysis.
}

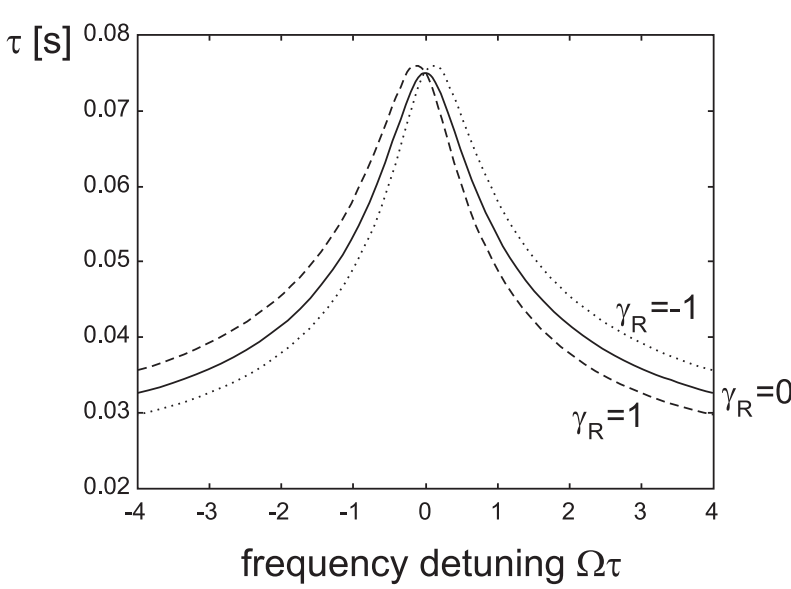

Figure 9. Dependence of time constant $\tau$ on $\Omega \tau$ with $\tau_{0}=75 \mathrm{~ms}$. Other parameters are $\gamma_{I} l=4$, feedback reflectivity $R=0.5$. Solid curve: $\gamma_{R}=0$; dashed curve $\gamma_{R}=1$, dotted curve $\gamma_{R}=-1$.

transverse wave numbers stayed constant for negative values of $\Omega \tau$. Since all values on the threshold curve $\gamma\left(k_{d} l\right)$ are a solution of the threshold equation, we calculated the values of $\gamma l$ for which the transverse wave number remained constant, as suggested by the experiment. These values differed from the ones determined by the absolute minima by only $5 \%$ for $\Omega \tau \geqslant-4$. A constant transverse wave number $k_{d} l$ is therefore obtainable without a large value of the coupling strength which is consistent with our experimental results.

It should be noted that the jump appearing in the transverse scale for certain negative frequency detunings $\Omega \tau$ is of a different kind compared to the jump appearing for large $n_{0} L / l$ when a second instability balloon takes over the absolute minimum of the threshold curves $[9,10]$. In our case, we have to deal with an intra-balloon competition, where a new global minimum is born at the expense of the old one which completely vanishes when going to larger $|\Omega \tau|$. This is the same type of jump as can be seen for the special case $\Omega=0$ for certain negative values of $n_{0} L / l$ (see figure 7 of [9]). Due to the special shape of the threshold curves, a whole band of transverse wave vectors can be excited and nonlinear interactions come into play. For the case considered in [9], the jump is accompanied by the appearance of a multiple pattern region with a large number of different transverse wave numbers which cannot be fully explained by the appropriate linear stability analysis, though good agreement between theory and experiment could be found outside this multiple pattern region. As a consequence, nonlinear interactions have to be taken into account for these special regions of parameters to give the exact value of the transverse scale of the pattern. Inclusion of nonlinear terms was considered in [10] and [24] for the special case $\Omega=0$. They complicate the theoretical analysis considerably and inclusion of these nonlinear contributions are therefore beyond the scope of this paper.

In figure $10(b)$, measurements and theoretical curves for a diffraction length $n_{0} L / l=0.25$ are depicted for different values of $\tau_{0}$. A qualitative agreement with the theoretical analysis can also be found here. Figure $10(c)$ shows the transverse wave number $k_{d} l$ as a function of the normalized diffraction length $n_{0} L / l$ for three values of $\Omega$ and $\tau_{0}=95 \mathrm{~ms}$. 

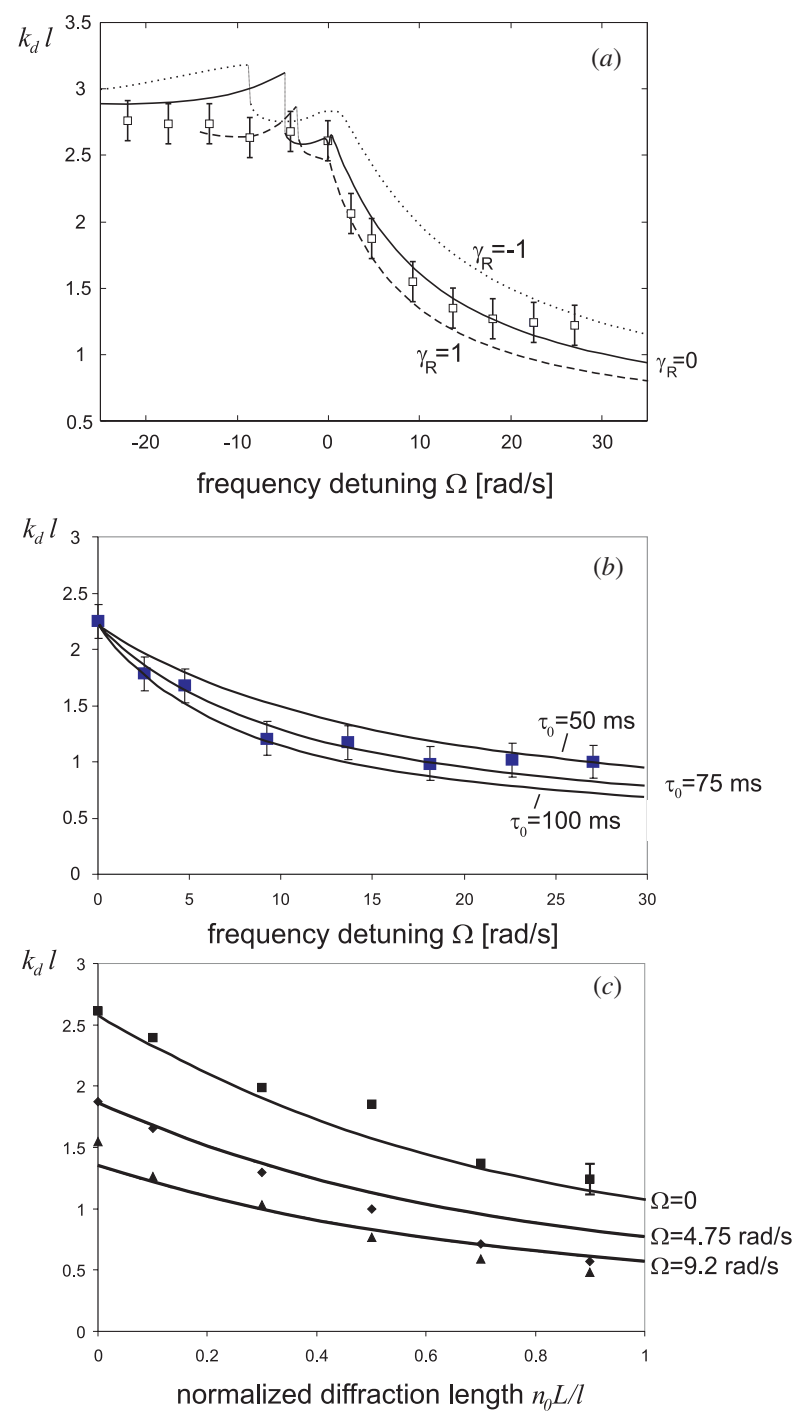

Figure 10. Comparison of theoretical and experimental results for the transverse wave number $k_{d} l$. (a) As a function of the frequency detuning for $n_{0} L / l=0$; solid curve: theory for $\gamma_{R}=0$; dashed curve: theory for $\gamma_{R}=1$; dotted curve: theory for $\gamma_{R}=-1$. All curves are given for a value of the time constant of $\tau_{0}=75 \mathrm{~ms}$ under consideration of the results depicted in figure 9. Squares: experimentally obtained values. (b) As a function of positive frequency detunings for $n_{0} L / l=0.25$, shown together with the theoretical curve for different values of $\tau_{0}$. (c) As a function of the diffraction length $n_{0} L / l$ for $\Omega=0$ (squares), $\Omega=4.75 \mathrm{rad} \mathrm{s}^{-1}$ (diamonds) and $\Omega=9.2 \mathrm{rad} \mathrm{s}^{-1}$ (triangles). Theoretical curves are given for a parameter value of $\tau_{0}=95 \mathrm{~ms}$.

Deviations between theory and experiment for larger diffraction lengths in figure $10(c)$ can be attributed to several effects. First, in our theoretical analysis, absorption is not taken into account in order to get an analytical threshold condition. The presence of absorption raises the experimental transverse wave numbers as shown in a numerical analysis for counterpropagating two-wave mixing with inclusion of absorption effects [20]. Second, to enable an analytical treatment of this problem, an intensity reflection coefficient of $R=1$ (compared to about $R=0.5$ in experiment) was assumed in the linear stability analysis. Another point to be considered is the intensity dependence of the grating relaxation parameter $\tau$. The experiment is performed with a Gaussian beam entering the crystal, i.e. we have to deal with a continuous distribution of time constants $\tau$ within the Gaussian beam.

We also measured the frequency detuning of the spatial sidebands $\delta$ by beating them interferometrically with the pump beam. The results of this measurement for $L=0$ are depicted in figure 11 showing that there indeed exists a frequency detuning in the spatial sidebands for positive values of $\Omega \tau$, but surprisingly not for $\Omega \tau<0$, in contrast to the theoretical predictions. For positive $\Omega \tau$, the opposing spot of the roll pattern in the optical far field showed a frequency detuning with a different sign and approximately the same value of $\delta$, which was a key assumption in our theoretical analysis. It is noteworthy here that the measurement of the beat frequency was performed for a small portion of the extended spatial sideband. In general, the sidebands carry a whole distribution of frequency shifts $\delta$, which is connected to the finite extension of the sideband. Let us assume that the extension of the sideband is $0.1 k_{d} l$ (bandwidth in Fourier space), which corresponds to a certain distribution of frequency detunings $\delta \tau$. We calculated this distribution for $\Omega \tau=0.15$ and found that a variation of $k_{d} l$ of $5 \%$ is transferred to a variation of $\delta \tau$ of approximately $20 \%$. The agreement between theory and experiment for positive values of $\Omega \tau$ is reasonable under these circumstances. We note that for negative frequency detunings $\Omega$, no significant frequency detuning of the spatial sideband could be measured in the experiment although it is predicted by our linear stability analysis. Obviously, we have to deal here with a static instability, $\delta=0$, although close inspection of the threshold condition (8) describing the linear stability curve of the system does not reveal any solutions for $\delta=0$ and nonzero $\Omega$. The theoretical curves for the sideband frequency detuning $\delta \tau$ as given in figure 11 clearly indicate the asymmetry between positive and negative $\Omega \tau$. However, the quantitative behaviour for negative values of $\Omega \tau$ could not be reproduced in the experiment. Even inclusion of an additional drift component $\gamma_{R}$ to the photorefractive coupling constant does not explain the static instability as observed in our experiment.

An additional factor to be noted is that an explicit intensity dependence of the grating relaxation parameter $\tau=\tau(I)$ was recently shown to have a possible influence on the type of instability in the counterpropagating photorefractive interaction with mirror boundary conditions [24]. For the special case $\Omega=0$ and $n_{0} L / l=0$, it was shown that all modes turned out to be stationary for $\tau(I) \sim I^{-1}$, whereas the threshold coupling strength and the mode with the fastest growth rate were not affected by the choice of an intensity dependent $\tau(I)$. On the other hand, the assumption $\tau=$ const leads to nonzero values of $\delta$ for a certain range of wave numbers $k_{d} l$. Nevertheless, the mode with the fastest growth rate is shown to be connected to $\delta=0$ even for the case $\tau=$ const, thus indicating a static instability as in the case $\tau(I)$. Although the type of instability was not changed in this special case, it is a reasonable assumption that for a special choice of parameters $n_{0} L / l$ and $\Omega \tau$ the intensity dependence of $\tau$ may have a direct influence on the type of instability and give rise to a static instability where a dynamic one is predicted by the linear stability analysis under the assumption $\tau=$ const as presented above. 


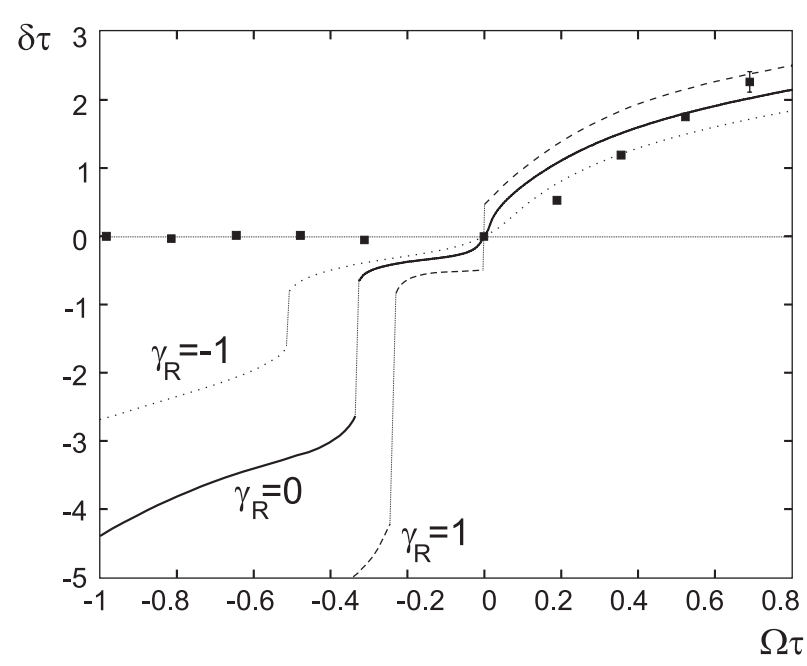

Figure 11. Theoretical and experimental results for the sideband frequency detuning $\delta \tau$ as a function of the pump frequency detuning $\Omega \tau$ for a parameter value of $n_{0} L / l=0$. Solid curve: theoretical values for $\gamma_{R}=0$; dashed curve: theory for $\gamma_{R}=1$; dotted curve: theory for $\gamma_{R}=-1$. The experimental values are given for a value of $\tau_{0}=75 \mathrm{~ms}$.

\section{Discussion and conclusion}

There is a similarity of effects connected to a frequency shift of the optical pump beams and those obtained for a tilted feedback mirror. A tilted feedback mirror is known to induce a drift instability which also results in a frequency detuning of the spatial sidebands as investigated in the case of an atomic nonlinearity in $[25,26]$. A linear dependence of the frequency detuning on the mirror tilt angle was also found and confirmed for the case of a photorefractive nonlinearity [8]. In both systems, a roll pattern develops carrying a frequency detuning $\delta$ and $-\delta$ in the opposing far field spots as analysed in [25] by Petrossian et al. They also reported a reduction of the transverse wave number for the case of a tilted mirror compared to the case of perpendicular incidence which is also the result of our experiment with a frequency-shifted collinear feedback beam. The difference, however, is that a frequency detuning effectively changes the complex coupling constant as can be seen in equation (19), whereas a tilted feedback beam $B \exp (\mathrm{i} b z$ ), with $b$ describing the angular deviations (see e.g. [27]), enters the two-wave mixing equations (1)-(3) in a more complicated manner. Nevertheless, both situations lead to a drift instability and frequency-shifted spatial sidebands. Self-imaging of the modulated pattern, which is a key assumption for a locked pattern, is no longer fulfilled in both cases. Self-reproduction after one round trip is prevented by the moving grating in one case and the angular deviation in the other case.

Finally, let us comment on the influence of a negative frequency detuning $\Omega \tau$ applied to the system. Experimentally, we found no significant changes in transverse scale and no frequency detuning of the spatial sideband thus indicating a fundamental asymmetry for positive and negative frequency detunings. A pattern locking appears to occur for negative frequency detunings $\Omega$, a phenomenon that has been seen for small mirror tilts in an experiment with atomic nonlinearity [26]. It is thus tempting to ascribe the disagreement between linear stability analysis and experiment for $\Omega<0$ to a nonlinear locking phenomena. However, for similar values of $|\Omega|$ we saw no evidence of locking for $\Omega>0$. This suggests that there is a different explanation of the asymmetry between positive and negative $\Omega$. While a full explanation of this asymmetry is still to be found we note that changing the sign of $\Omega$ changes the character of the nonlinearity. Starting with equations (1)-(3) and including an effective dark intensity contribution $I_{d}$ due to thermal excitation of charge carriers in the denominator of the grating equation (3), one can show that the nonlinear part of the refractive index $\Delta n$ can be written as

$$
\Delta n \sim \frac{\gamma_{R}+\gamma_{I} \Omega \tau}{\left(1+\Omega^{2} \tau^{2}\right)\left(2+I_{d} / I\right)}
$$

with $I=|F|^{2}=|B|^{2}$ denoting the beam intensity. Our experimental choice of crystal and its orientation yields $\gamma_{R} \ll$ 1 , and $\gamma_{I}>0$. This indicates that $\Omega>0$ corresponds to selffocusing, whereas $\Omega<0$ gives a self-defocusing effect. There is thus a qualitative difference in the nonlinearity depending on the sign of $\Omega$ although the detailed mechanism behind the clamping of the pattern scale and sideband frequency shift observed for negative $\Omega$ remains unknown. This deviation between theory and experiment is currently under investigation.

In conclusion, we have shown that pattern manipulation is possible by frequency detuning one of the beams entering the nonlinear medium. By performing a single feedback mirror experiment with a photorefractive nonlinearity, we obtained a switch from a square to a hexagonal and from a squeezed hexagonal to a rectangular structure thus demonstrating the principle function of this method. We derived an analytical expression for the onset of modulational instability in the case of frequency detuned pump beams counterpropagating in a photorefractive material with mirror boundary conditions. A frequency detuning of the pumps was shown to lower the threshold for modulational instability and the transverse wave number. The measurements of the spatial scale could be shown to be in reasonable agreement with the results obtained from the linear stability analysis. A positive frequency detuning of the pumps is always connected to a drift instability, resulting in a frequency detuning of the spatial sidebands. Appearance of vortices in the spatial sidebands in this case was also reported. Our experimental system shows an asymmetry in $\Omega$, since negative pump frequency detunings had nearly no influence on the transverse wave number. No sideband frequency detunings could be found in this parameter regime, also sideband intensity fluctuations and oscillating appearance of vortices were not seen. However, our experiment indicates that in special parameter regions, both positive and negative pump frequency detunings enable to manipulate the geometry of the output of our pattern forming system. We believe that this constitutes a generic feature of pattern forming nonlinear optical systems.

\section{Acknowledgments}

M Schwab and C Denz gratefully acknowledge kind support by Professor T Tschudi. M Schwab acknowledges financial support from the FAZIT-Stiftung and the University of Wisconsin. 


\section{References}

[1] Lugiato L A, Brambilla M and Gatti A 1999 Adv. Atom. Mol. Opt. Phys. 40229 ed B Bederson and H Walther

[2] Arecchi F T, Boccaletti S and Ramazza P 1999 Phys. Rep. 3181

[3] Petrossian A, Pinard M, Maitre A, Courtois J-Y and Grynberg G 1992 Europhys. Lett. 18689

[4] MacDonald R and Eichler H J 1992 Opt. Commun. 89289

[5] Thüring B, Neubecker R and Tschudi T 1993 Opt. Commun. 102111

[6] Honda T 1993 Opt. Lett. 18598

[7] Honda T and Banerjee P P 1996 Opt. Lett. 21 779-81

[8] Denz C, Schwab M, Sedlatschek M, Tschudi T and Honda T 1998 J. Opt. Soc. Am. B 152057

[9] Schwab M, Denz C and Saffman M 1999 Appl. Phys. B 69429

[10] Lushnikov P M 1998 Zh. Éksp. Teor. Fiz. 1131122

[11] Vorontsov M A and Miller W B (ed) 1995 Self-Organization in Optical Systems and Applications in Information Technology (Berlin: Springer)

[12] Mamaev A V and Saffman M 1998 Phys. Rev. Lett. 803499

[13] Ackemann T, Giese B, Schäpers B and Lange W 1999 J. Opt. B: Quantum Semiclass. Opt. 170

[14] Schwab M, Saffman M, Denz C and Tschudi T 1999 Opt. Commun. 170129

[15] Martin R, Scroggie A J, Oppo G-L and Firth W J 1996 Phys. Rev. Lett. 774007
[16] Harkness G K, Oppo G-L, Benkler E, Kreuzer M, Neubecker R and Tschudi T 1999 J. Opt. B: Quantum Semiclass. Opt. 1114

[17] Yeh P 1993 Introduction to Photorefractive Nonlinear Optics, (New York: Wiley)

[18] Hodgson N and Weber H 1997 Optical Resonators (London: Springer)

[19] Arecchi F T, Giacomelli G, Ramazza P L and Residori S 1990 Phys. Rev. Lett. 652531

[20] Sandfuchs O, Kaiser F and Belić M R 1998 J. Opt. Soc. Am. B 152070

[21] Schwab M, Denz C and Saffman M 2001 J. Opt. Soc. Am. B 18628

[22] Chernykh A I, Sturman B I, Aguilar M and Agulló-López F 1997 J. Opt. Soc. Am. B 141754

[23] Günter P and Huignard J P (ed) 1998 Photorefractive Materials and their Applications I (Berlin: Springer)

[24] Sandfuchs O, Kaiser F and Belić M R 2001 J. Opt. Soc. Am. B 18505

[25] Petrossian A, Dambly L and Grynberg G 1995 Europhys. Lett. 29209

[26] Seipenbusch J P, Ackemann T, B. Schäpers, Berge B and Lange W 1997 Phys. Rev. A 56 R4401

[27] Denz C, Goltz J and Tschudi T 1989 Opt. Commun. 72129 